\title{
ORIGINAL ARTICLE Obesity reduces the pro-angiogenic potential of adipose tissue stem cell-derived extracellular vesicles (EVs) by impairing miR-126 content: impact on clinical applications
}

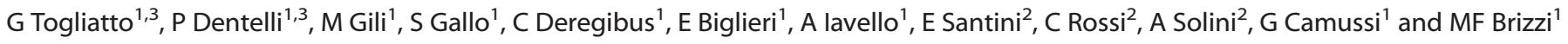

BACKGROUND/OBJECTIVES: Soluble factors and cell-derived extracellular vesicles (EVs) are crucial tissue repair mediators in cellbased therapy. In the present study, we investigate the therapeutic impact of EVs released by adipose tissue-derived stem cells (ASCs) recovered from obese subjects' visceral and subcutaneous tissues.

METHODS: ASCs were recovered from 10 obese (oASCs) and 6 non-obese (nASCs) participants and characterized. In selected experiments, nASCs and oASCs were cultured with palmitic acid (PA) or high glucose (HG), respectively. EVs from obese (oEVs) and non-obese (nEVs) subjects' visceral and subcutaneous ASCs were collected after ultracentrifugation and analyzed for their cargo: microRNA-126 (miR-126), vascular endothelial growth factor (VEGF), and matrix metalloproteinase 2 (MMP-2), and for their biological effects on endothelial cells (ECs). Western blotting analysis and loss- and gain-of function experiments were performed. RESULTS: oEVs show impaired angiogenic potential compared with nEVs. This effect depends on EV cargo: reduced content of VEGF, MMP-2 and, more importantly, miR-126. We demonstrate, using gain- and loss-of-function experiments, that this reduced miR-126 content leads to Spred1 upregulation and the inhibition of the extracellular signal-regulated kinase 1/2 mitogen-activated protein kinase pathway in ECs. We also show that PA treatment of nASCs translates into the release of EVs that recapitulate oEV cargo. Moreover, HG treatment of oASCs further reduces miR-126 EV content and EV-mediated in vitro angiogenesis. Finally, impaired pro-angiogenic potential is also detected in EVs released from obese subcutaneous adipose tissue-derived ASCs.

CONCLUSIONS: These results indicate that obesity impacts on EV pro-angiogenic potential and may raise concerns about the use of adipose tissue-derived EVs in cell-based therapy in the obese setting.

International Journal of Obesity (2016) 40, 102-111; doi:10.1038/ijo.2015.123

\section{INTRODUCTION}

Obesity is a pandemic health problem. ${ }^{1}$ In particular, visceral fat accumulation results in adipocyte and adipose tissue function abnormalities leading to a plethora of diabetogenic and atherogenic abnormalities, which often directly contribute to cardiovascular disease. ${ }^{2}$ Adipose tissue is connective tissue specializes in accumulating fat into adipocytes. Besides adipocytes, adipose tissue also contains mesenchymal stem cells (MSCs), denoted as adipose-derived stem cells (ASCs). ${ }^{3-5}$ Similarly to stem cells present in other different adult tissues, ASCs are multi-potent cells that are able to differentiate into mesenchymal tissues. ${ }^{6}$ The fact that ASCs act as an important source of cells for tissue regeneration and cell-based therapy means that they have attracted particular interest. ${ }^{7}$ Although ASCs have been shown to participate in tissue repair, their precise mechanism of action is still open to question. It is generally accepted that their most relevant mechanism proceeds via paracrine actions. ${ }^{8-10}$ A large amount of data has more recently indicated that ASCs use membrane-derived small vesicles, denoted as cell-derived extracellular vesicles (EVs), as well as the secretion of soluble factors, to repair damaged tissues. ${ }^{11,12} \mathrm{EVs}$, including exosomes and microvesicles, are shed from many cell types and have been shown to have important roles in cell-cell communication. ${ }^{13,14}$ It has also been shown that EVs can release various active molecules, including signaling molecules, mRNAs and microRNAs (miRs), in both physiological and pathological settings. ${ }^{15}$ Recent in vitro and in vivo studies have demonstrated that EVs have a relevant role in inflammatory diseases, including atherosclerosis, by transferring pro-angiogenic factors, such as growth factors (vascular endothelial growth factor (VEGF), platelet-derived growth factor (PDGF)), and matrix metalloproteinases (MMP-2, MMP-9) into target cells. ${ }^{16}$ Data on the pro-angiogenic potential of endothelial progenitor cell-derived EVs in a mouse model of hind limb ischemia have been provided. ${ }^{17}$ Moreover, it has also been shown that MSCderived MVs can protect against ischaemia-reperfusion-induced acute and chronic kidney injury. ${ }^{18-20}$ Finally, the healing properties of miRs that are carried by MSC-derived EVs have also been provided. $^{21}$

The activation of cells by chemical and physical stimuli, including hypoxia and shear stress, is associated with EV release. $^{22}$ In fact, circulating EVs are more abundant in a number of clinical settings, including diabetes and cardiovascular disease. $^{23,24}$ An important feature of EVs is their heterogenic cargo. Indeed, EV cargo can vary according to challenges by different stimuli, while differing cell types challenged with the same stimulus can release EVs with different cargo. ${ }^{22}$ Although general EV functions have been the topic of recent studies in various pathological settings, no data are currently available on EV

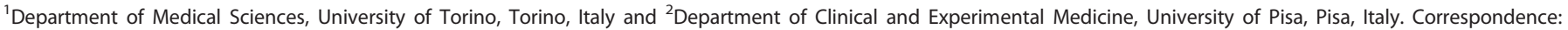
Professor G Camussi or Professor MF Brizzi, Department of Medical Sciences, University of Torino, Corso Dogliotti 14, Torino 10126, Italy.

E-mail: giovanni.camussi@unito.it or mariafelice.brizzi@unito.it

${ }^{3}$ These authors contributed equally to this work.

Received 20 January 2015; revised 18 May 2015; accepted 22 June 2015; accepted article preview online 30 June 2015 ; advance online publication, 21 July 2015 
functional activity in an obese setting. Furthermore, it is still unknown whether ASCs derived from visceral/subcutaneous adipose tissue in obese patients can release EVs, which carry different components than those released by non-obese subjects. This would be of particular interest given their potential clinical impact. The present study analyzes the EV release and cargo in adipose tissue-derived ASCs of obese non-diabetic patients, paying particular attention to their pro-angiogenic activity.

\section{MATERIALS AND METHODS}

Reagents and antibodies are reported in Supplementary Table S1 of Supplementary Information.

\section{Patients and controls}

A total of 10 obese and 6 non-obese subjects (controls) who underwent abdominal surgery in our clinic were included in the study. Clinical characteristics are reported in Supplementary Table S2. Ethics approval was obtained from the Institutional Review Board of S. Giovanni Battista Hospital, Turin, Italy. Informed consent was obtained from all patients according to the Helsinki Declaration. We also declare that we had no direct contact with the participants in the present study (patients' anonymity was guaranteed).

Isolation of human visceral and subcutaneous-derived ASCs

Paired human visceral and subcutaneous adipose tissue samples were recovered from the above reported subjects (obese (oASC); non-obese (nASC)) and processed as previously described. ${ }^{6}$ Freshly isolated ASCs were characterized for their surface expression profile by fluorescence-activated cell sorting (FACS) (CD29, CD105, CD44, CD90, CD73) and for Oct4 content by western blotting analysis. In selected experiments, nASCs were cultured for 2 days with $300 \mathrm{umolI}^{-1}$ of palmitic acid (PA). ${ }^{25}$ Last day, nASCs were maintained with PA, without fetal bovine serum, and processed for EV isolation. Separately, EVs were obtained from OASCs and maintained in culture with high glucose (HG) concentration $\left(25 \mathrm{mmolI}^{-1}\right)$. In selected experiments nASCs and oASCs were cultured in Dulbecco's modified Eagle's medium (DMEM) $+10 \%$ fetal bovine serum $+0.5 \mathrm{mmol} \mathrm{I}^{-1} 3$-isobutyl1-methylxanthine $+0.25 \mathrm{mmoll}^{-1}$ dexamethasone $+1 \mathrm{mg} \mathrm{ml}^{-1}$ insulin (all Sigma-Aldrich, St Louis, MO, USA) to stimulate adipocyte differentiation.

\section{Cell cultures}

Endothelial cells (ECs) isolated as previously described ${ }^{26}$ were cultured in M199 without bovine calf serum for $24 \mathrm{~h}$ in the presence of EVs recovered from ASCs, which were treated as indicated. Human ASCs (positive controls; Lonza, Basel, Switzerland) were cultured in the specific Lonza medium. The NIH 3T3L1 cell line (from ATCC, Middlesex, UK) was cultured in adipogenic differentiating medium.

\section{Isolation of ASC-derived EVs}

oASCs and nASCs from both visceral and subcutaneous tissues were cultured in DMEM, without fetal bovine serum, for $24 \mathrm{~h}$ in order to collect EVs from supernatants. After being centrifuged at $3000 \mathrm{~g}$ for $30 \mathrm{~min}$ to remove debris, cell-free supernatants were submitted to differential ultracentrifugation at 10000 and $100000 \mathrm{~g}$ (Beckman Coulter Optima L-90K ultracentrifuge; Beckman Coulter, Fullerton, CA, USA) for $3 \mathrm{~h}$ at $4{ }^{\circ} \mathrm{C}$. EVs were used freshly or stored at $-80^{\circ} \mathrm{C}$ after re-suspension in DMEM, which was supplied with $5 \%$ dimethyl sulfoxide. ${ }^{27,28}$ Frozen EVs were washed and pelleted by $100000 \mathrm{~g}$ ultracentrifugation to remove dimethyl sulfoxide before cellular experiments. No difference in biological activity was observed between fresh and stored EVs. The protein content of EVs was quantified using the Bradford method (Bio-Rad, Hercules, CA, USA). Any possible contamination was tested using a Limulus amebocyte assay (concentration $<0.1 \mathrm{ng} \mathrm{ml}^{-1}$ ). EV size distribution analysis was performed using a NanoSight LM10 (NanoSight Ltd, Minton Park, UK). The particles in the samples were illuminated using a laser light source, and the scattered light was captured by camera and analyzed using Nanoparticle Tracking Analysis. Nanoparticle Tracking Analysis automatically tracked and sized particles according to Brownian motion and the diffusion coefficient. Results were displayed as a frequency size distribution graph and were outputted to a spreadsheet. In selected experiments, EVs were treated with $5 \mathrm{U}$ RNAse for $3 \mathrm{~h}$ at $37^{\circ} \mathrm{C}$; the reaction was stopped by $10 \mathrm{U} \mathrm{ml}^{-1}$ RNAse inhibitor (Ambion, Carlsbad, CA, USA) and EVs were washed using ultracentrifugation. ${ }^{18}$

\section{Characterization of ASC-derived EVs}

FACS analysis of ASC-derived EVs was performed as indicated ${ }^{19}$ using the following fluorescein isothiocyanate- or phycoerythrin-conjugated antibodies: CD29, CD105, CD44, CD90, CD73, CD81. Fluorescein isothiocyanate or phycoerythrin mouse non-immune isotypic immunoglobulin $\mathrm{G}$ (BD Biosciences, Franklin Lakes, NJ, USA) was used as control. Briefly, FACS analysis of ASC-derived EVs was performed using a Guava easyCyte Flow Cytometer (Millipore, Darmstadt, Germany). Fluorescein isothiocyanate- or phycoerythrin-conjugated antibodies were added to a suspension of EVs (250 particles $\mu l^{-1}$ in $100 \mu \mathrm{l}$ ) for $15 \mathrm{~min}$ at $4^{\circ} \mathrm{C}$ to perform FACS analysis of EVs. The volume was increased with a FACS flow up to $500 \mu \mathrm{l}$, and the expression of surface markers was evaluated. CD63 content in EVs was analyzed by western blotting

\section{EV internalization by ECs}

The internalization of EVs into ECs was evaluated using confocal microscopy (LSM5-PASCAL; Zeiss, Oberkochen, Germany). A pool of approximately $5 \times 10^{9} \mathrm{EV}$ particles was labeled with red fluorescent PKH26 dye $\left(2 \mu \mathrm{ml}^{-1}\right)$ for $30 \mathrm{~min}$ at $37^{\circ} \mathrm{C}$, and then EVs were washed and ultracentrifuged at $100000 \mathrm{~g}$ for $1 \mathrm{~h}$ at $4^{\circ} \mathrm{C}$. EV pellets were suspended in DMEM and added to ECs $\left(1 \times 10^{6}\right)$ for several hours in order to detect their internalization (photomicrographs reported in the results show EV internalization after $1 \mathrm{~h}$ ). EVs were preincubated with blocking monoclonal antibody $\left(1 \mu \mathrm{g} \mathrm{ml}^{-1}\right.$ CD29 (BD Pharmingen, Franklin Lakes, NJ, USA) in the selected experiments. ${ }^{29}$

\section{Western blotting analysis}

Cells and EVs were lysed and protein concentrations were obtained as previously described..$^{30}$ In all, $50 \mu \mathrm{g}$ proteins for cells and $10 \mu \mathrm{g}$ for EVs were subjected to sodium dodecyl sulfate-polyacrylamide gel electrophoresis. Details are reported in Supplementary Information.

\section{RNA isolation and quantitative real-time PCR}

Total RNA was isolated from nASCs and oASCs using the TRlzol reagent (Invitrogen, Carlsbad, CA, USA) and from different EVs using the mirVana RNA Isolation Kit (Ambion), according to the manufacturer's instructions. RNA was quantified spectrophotometrically (Nanodrop ND-1000, Nanodrop Technologies, Wilmington, DE, USA), because intact $18 \mathrm{~S}$ and $28 \mathrm{~S}$ rRNAs were difficult to detect in the EVs. RNA from cells and EVs was then reverse-transcribed using a TaqMan microRNA RT Kit, specific for miR-126 and miR-130a, and subjected to quantitative real-time PCR as detailed in Supplementary Information. Gain- and loss-of-function experiments were performed as detailed in Supplementary Information. EV isolation was then obtained from nASCs that had been depleted of miR-126.

Tube-like structure formation (in vitro angiogenesis assay) Tube-like structure formation from ECs treated with or without VEGF $\left(10 \mathrm{ng} \mathrm{ml}^{-1}\right)$ and various EVs recovered from nASCs or oASCs was analyzed as described ${ }^{30}$ and detailed in Supplementary Information.

Migration assay and cell proliferation and adhesion assay are detailed in Supplementary Information.

\section{Senescence assay}

Senescence was evaluated by measuring acidic $\beta$-gal activity on nASCs, nASCs+PA and oASCs. ${ }^{31}$ Details are reported in Supplementary Information.

\section{Statistical analysis}

All data are presented as mean or percentage \pm s.e.m. The D'AgostinoPearson test was used to test normality. Data on biometric measurements of patients and controls, on the in vitro angiogenic, migration, adhesion and senescence assays, on miR expression, cell proliferation, loss- and gainof-function experiments and finally on densitometric analysis for western blots were analyzed using Student's $t$-tests for two-group comparison and using one-way analysis of variance, followed by Tukey's multiple comparison test, for $\geqslant 3$ groups. Three experiments performed in triplicate were the minimum sample size ensuring $90 \%$ statistical power between 
experimental groups, with a probability level of 0.05 , two-tailed hypothesis. The cutoff for statistical significance was set at $P<0.05$ $\left({ }^{*} P<0.05,{ }^{* *} P<0.01,{ }^{* * * P} P<0.001\right)$. All statistical analyses were carried out using GraphPad Prism version 5.04 (GraphPad Software, Inc., La Jolla, CA, USA).

\section{RESULTS}

ASC isolation from obese subjects and controls

Although both subcutaneous and visceral adipose tissues are potential sources of adult stem cells, ${ }^{32}$ subcutaneous adipose tissue has mostly been used in investigations into their potential clinical applications. ${ }^{32,33}$ Visceral adipose tissue abundance in pathological settings, which is connoted by drastic changes in visceral adipose mass, ${ }^{32,33}$ has led us to evaluate its relevance in providing ASCs or derivates for clinical purposes. ASCs were thus isolated from visceral adipose tissues that had been recovered from 10 obese patients $\left(\mathrm{BMI}>30 \mathrm{Kg} \mathrm{m}^{-2}\right)$ and from 6 non-obese subjects, used as controls (BMI $<30 \mathrm{Kg} \mathrm{m}^{-2}$ ), who all underwent abdominal surgery. ${ }^{6}$ Clinical characteristics of the participants are summarized in Supplementary Table S2. ASC availability was evaluated in obese (oASCs) subjects and controls (nASCs). There were no differences in the number of ASCs between the two groups (mean number of cells $\times 10^{3}$ per $\mathrm{g}$ fat tissue: $60.03 \pm 8.10$ s.e.m. oASCs, 10 samples; $58.9 \pm 7.4$ s.e.m. nASCs, 6 samples). Oct4 expression and CD markers were used for stem cell characterization in both obese patients and controls (Figures $1 \mathrm{a}$ and $\mathrm{c}$ ). As reported in Supplementary Figures S1A and $\mathrm{C}$, oASCs show a reduced proliferation rate and an increased number of senescent cells while preserving their adipocyte differentiating capability (peroxisome proliferator-activated receptor- $\gamma 2$ expression).

\section{Characterization of ASC-derived EVs}

It has widely been reported that ASCs can release EVs. ${ }^{28}$ We herein investigate whether differences in $\mathrm{EV}$ release can be detected when visceral ASCs are derived from non-obese (nEVs) and obese (oEVs) subjects. Figures $1 \mathrm{f}$ and $\mathrm{g}$ shows that no differences in terms of EV number and size were detected by Nanosight. EV
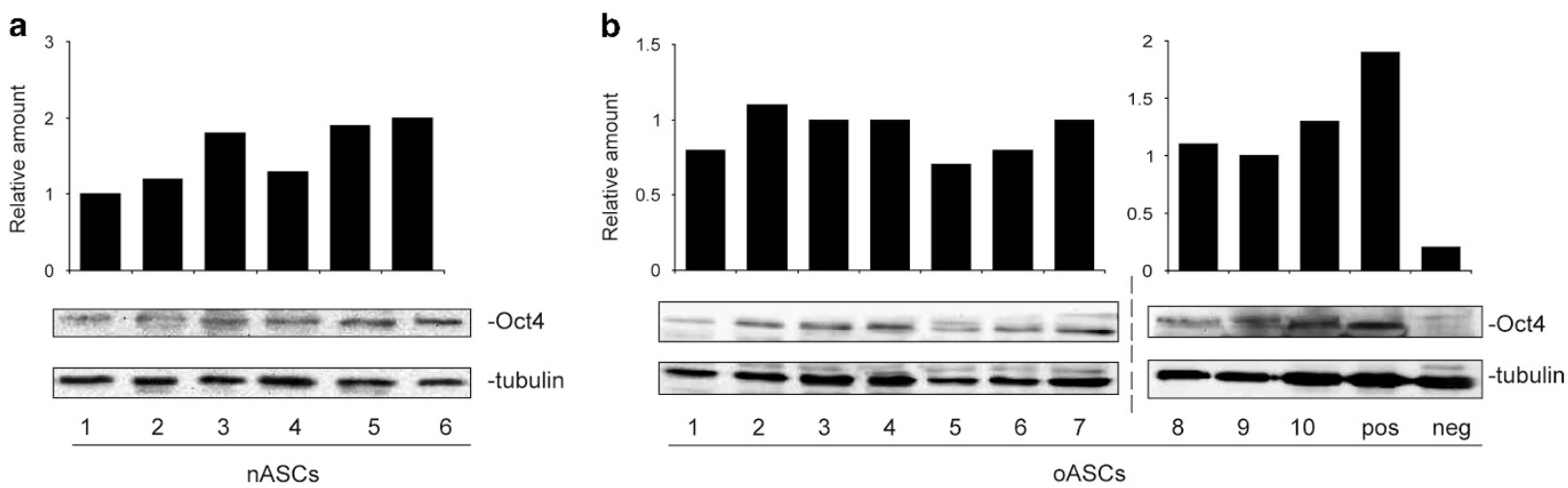

C

\begin{tabular}{|c|c|c|}
\hline & nASCs & oASCs \\
\hline CD29 & $54 \pm 5$ & $80 \pm 4$ \\
\hline CD44 & $89 \pm 3$ & $90 \pm 5$ \\
\hline CD105 & $78 \pm 4$ & $83 \pm 6$ \\
\hline CD90 & $81 \pm 5$ & $86 \pm 3$ \\
\hline CD73 & $85 \pm 6$ & $87 \pm 3$ \\
\hline
\end{tabular}

\begin{tabular}{|c|c|c|}
\hline & $\mathrm{nEVs}$ & oEVs \\
\hline $\mathrm{CD} 29$ & $50 \pm 4.2$ & $45 \pm 4.8$ \\
\hline CD44 & $60 \pm 3.3$ & $48 \pm 5.3$ \\
\hline CD105 & $49 \pm 3$ & $37 \pm 4$ \\
\hline CD90 & $75 \pm 8.1$ & $80 \pm 7.4$ \\
\hline $\mathrm{CD} 73$ & $20 \pm 1.5$ & $14 \pm 2.6$ \\
\hline CD81 & $57 \pm 2.8$ & $52 \pm 3.6$ \\
\hline
\end{tabular}
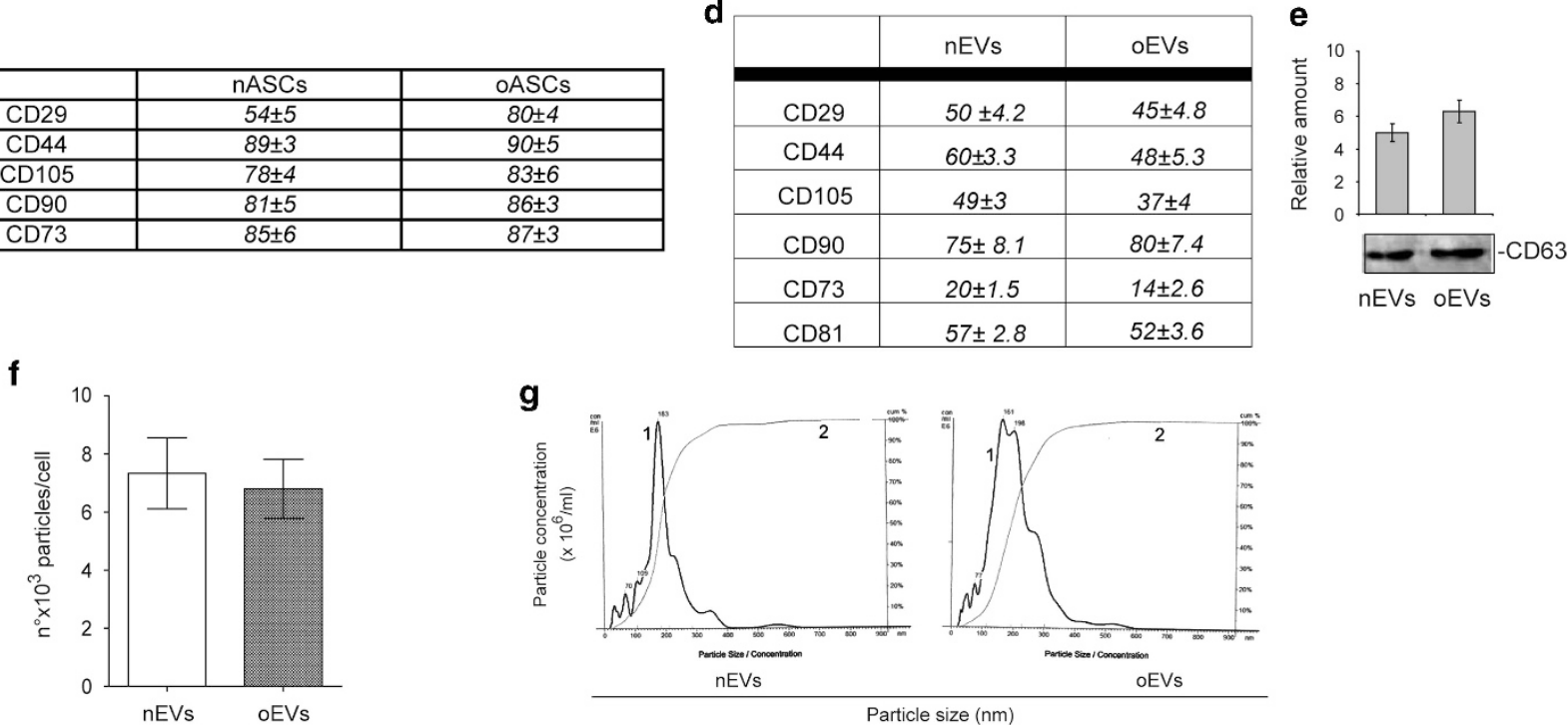

Figure 1. Characterization of ASCs and ASC-derived EVs recovered from obese subjects and non-obese controls. (a, b) Cell extracts of visceral adipose tissue samples recovered from obese subjects (oASCs, $n=10$ ) and non-obese subjects $(n A S C s, n=6)$ were analyzed by western blotting for Oct4 content by densitometry. Protein levels were normalized to tubulin content. pos: positive control, human ASCs purchased from Lonza; neg: negative control, adipocytes derived from NIH 3T3L1 cell line. (c, d) Table resuming flow cytometry analysis of nASC, oASC and EV surface markers (CD29, CD44, CD105, CD90 and CD73 for ASCs and EVs and CD81 for EVs, expressed as mean \pm s.e.m.). (e) EV marker CD63 showed by western blotting analysis in nEVs and oEVs derived from nASCs $(n=6)$ and oASCs $(n=10)$. (f) Number of EV particles (mean \pm s.e.m.) per cell at isolation. Data refer to EVs from visceral adipose tissue samples recovered from obese subjects (oEVs, $n=10$ ) and non-obese subjects (nEVs, $n=6$ ). (g) Representative images of a NanoSight analysis performed on the 100k fraction of ASC-derived EVs. Curve 1 , relationship between particle distribution (left $y$ axis) and particle size ( $x$ axis); curve 2, correlation between cumulative percentage distribution of particles (percentile in right $y$ axis) and particle size ( $x$ axis). 
mesenchymal origin was demonstrated by analyzing their surface markers (Figures $1 \mathrm{~d}$ and e). The pro-angiogenic potential of EVs has been provided. ${ }^{17,28}$ In order to investigate whether oEVs can be exploited for angiogenic purposes, potential EV pro-angiogenic capability was evaluated by assaying VEGF and MMP-2 content. Results are reported in Figure $2 a$ and clearly demonstrate that the content of both VEGF and MMP-2 was lower in oEVs than in nEVs. Unexpectedly, we found that in OASCS VEGF and MMP-2 content was increased (Supplementary Figure S1D). Moreover, as EVs are capable of releasing various active molecules including miRs, we decided to evaluate the EV content of pro-angiogenic miRs, miR-126 and miR-130a. Data reported in Figure $2 \mathrm{~b}$ demonstrate that miR-126 content, but not miR-130a, was reduced in oEVs despite no significant differences detected in the cells (Supplementary Figure S1E).

Thus data indicate that oEVs may be less effective in inducing pro-angiogenic signals.

a

b

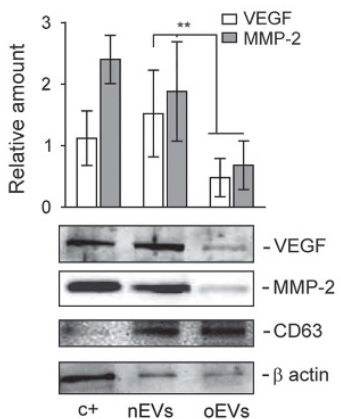

miR-126 expression
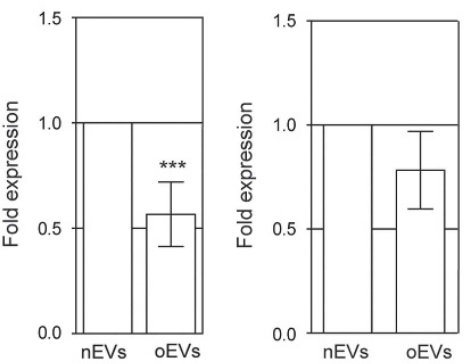

$\mathrm{nEV}$ and oEV internalization and EC biological effects

EVs have recently been the focus of increased levels of attention in their role as well-preserved evolutionary cell-to-cell communication mechanisms. ${ }^{13,14}$ In this regard, internalized EVs are known to modulate vessel formation by inducing angiogenesis or promoting EC survival. ${ }^{17,34}$ Thus, nEVs and oEVs that are labeled with PKH26 dye were evaluated for incorporation into target cells. nEVs and oEVs were incorporated by ECs as shown in Figure 2c. Moreover, we show that this internalization depends on the presence of the CD29 surface marker (Figure 2c). Functional studies were performed to evaluate the biological relevance of EV internalization into ECs. To this end, we analyzed the ability of $\mathrm{nEVs}$ and oEVs to induce EC migration and tube-like structure formation. The results reported in Figures $2 \mathrm{~d}$ and e demonstrate that oEVs are impaired in their ability to induce migration and angiogenesis in vitro as compared with nEVs. No significant reduction in the number of viable cells was detected.
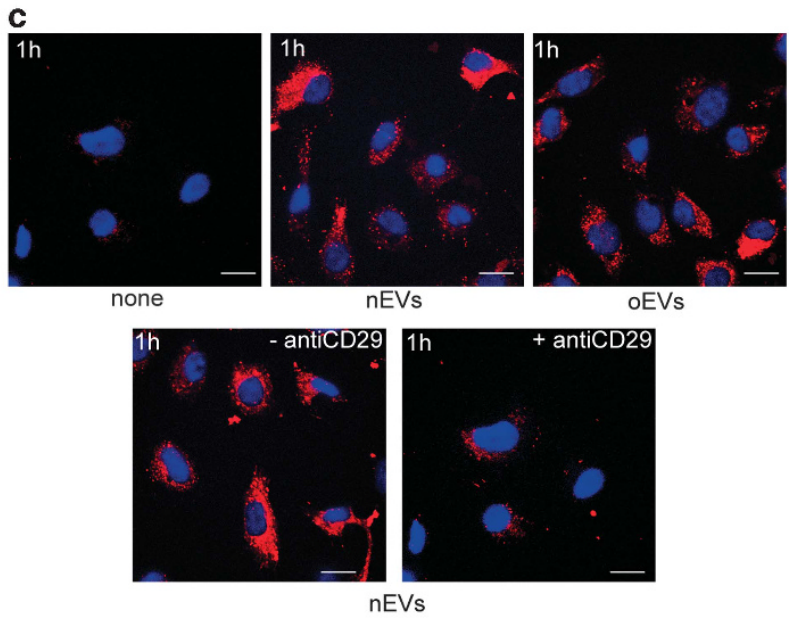

d In vitro angiogenesis

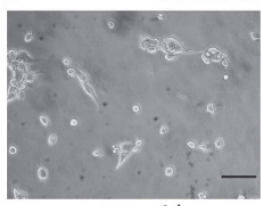

neg ctrl

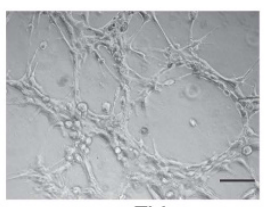

$\mathrm{nEVs}$

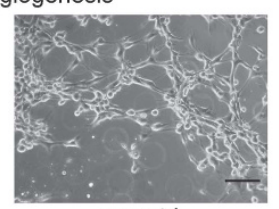

pos ctrl

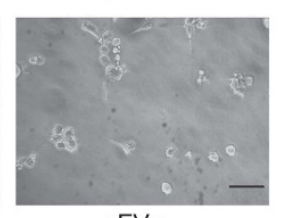

oEVs e

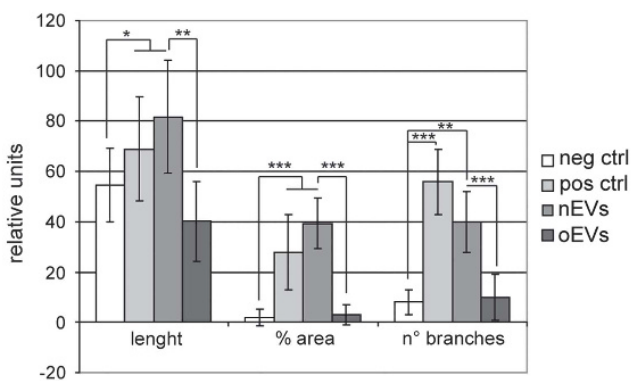

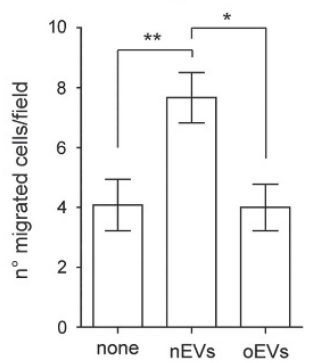

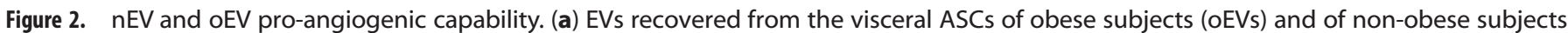
(nEVs) were analyzed by western blotting for VEGF and MMP-2 content and normalized to CD63 and $\beta$-actin content. IL-3-treated ECs were used as a positive control $(c+)$. The results are representative of all nEVs and oEVs samples ( $n=6$ and $n=10$, respectively) $(* * P<0.01$ for MMP-2 and VEGF content in oEVs vs nEVs). (b) miR-126 and miR-130 expression was evaluated by quantitative real-time PCR on nEVs and oEVs recovered from $\mathrm{nASCs}$ and oASCs, respectively. Data normalized to RNU6B are representative of all samples ( $\mathrm{nEV} s, n=6$; oEVs, $n=10$ ) (*** $P 0.001$ oEVS vs nEVs). (c) Representative images obtained by confocal microscope of ECs treated for $1 \mathrm{~h}$ without (none) or with PHK26-

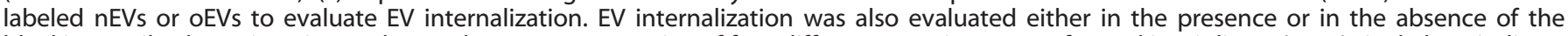
blocking antibody against CD29. The results are representative of four different experiments performed in triplicate ( $n=4)$. Scale bars indicate

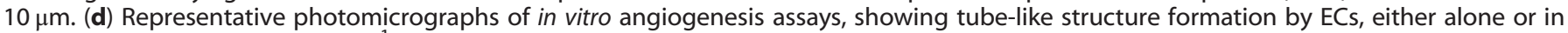
the presence of VEGF (10 ng ml ${ }^{-1}$ ) (positive control) or nEVs and oEVs. Quantitative analysis of the number and length of branches and percentage of vessel area (\% area) of in vitro formed vessel-like structures is reported as mean \pm s.e.m. The results are representative of four different experiments performed in triplicate $(n=4)$ (for length, ${ }^{*} P<0.05$ pos ctrl and nEVs vs neg ctrl; ${ }^{* *} P<0.01$ oEVs vs nEVs; for $\%$ area, ${ }^{* * *} P<0.001$ pos ctrl and nEVs vs neg ctrl, oEVs vs nEVs; for no. of branches, ${ }^{* * *} P<0.001$ pos ctrl vs neg ctrl, ${ }^{* *} P<0.01$ nEVs vs neg ctrl,

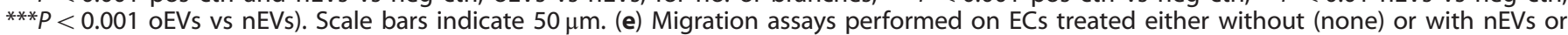
oEVs. The results are representative of four different experiments performed in triplicate $(n=4)\left(* * P<0.01, \mathrm{nEVs}\right.$ vs none, ${ }^{*} P<0.05$ oEVs vs nEVs). 
oEV functional derangements depend on cargo

Particular attention has been paid to the horizontal transfer of functional RNAs by EVs, ${ }^{8,9,32}$ and so in order to investigate the possibility that $n E V$ functional activity depends on RNA cargo, $\mathrm{nEV}$ s were pretreated with RNAse and evaluated for their ability to induce migration and tube-like structures. As shown in Figures $3 a$ and $b$, while RNAse-pretreated nEVs were not able to induce EC migration or angiogenesis in vitro, they are still able to induce vascular cell adhesion molecule-1 expression resulting in mononuclear cell adhesion (Figures $3 \mathrm{c}$ and d). miR-126's pro-angiogenic properties prompted us to investigate whether miR-126 contributes to EV-mediated biological function. $\mathrm{nEV}$ s that had been obtained after nASCs were treated with miR-126 antagomir (Supplementary Figures S2A and B) were used in the biological assay. Again, this treatment prevents nEV biological activity (Figures $3 a$ and $b$ ). To further validate the biological relevance of miR-126 in $\mathrm{nEV}$ function, ECs were transfected with pre-miR-126, and oEVs were used in the biological assays. Unlike observations in ECs transfected with the scramble control, a partial improvement in migration and tube-like formation was detected in ECs that overexpress miR-126 (Figures 3e and f). The reduced VEGF content in oEVs led us to evaluate whether the addition of VEGF may be more effective in mediating migration and tube-like structure formation than miR-126 overexpression alone. Indeed, we found that migration and tube-like structure formation was almost completely recovered when ECs that overexpress miR-126 were also stimulated with VEGF (Figures $3 e$ and f). These data indicate that reduced miR-126 and VEGF content impact on oEV biological action.

Reduced miR-126 expression in oEVs increases the content of Spred 1 and interferes with extracellular signal-regulated kinase 1/2 (Erk1/2) mitogen-activated protein kinase (MAPK) activation The miR-126 pro-angiogenic effect was originally correlated with its suppression of Spred1 expression and the inhibition of the MAPK signaling pathway. ${ }^{35}$ Spred1 content and Erk1/2 MAPK activation were thus evaluated in ECs that had been challenged with $n E V s$ and oEVs. nEVs were able to reduce Spred1 content resulting in Erk1/2 MAPK activation (Figures $4 a$ and b), which is consistent with increased miR-126 expression. As expected, Spred 1 content increased and Erk1/2 activation decreased when oEVs were used to stimulate ECs (Figures $4 a$ and b). Similar results were obtained when miR-126-depleted nEVs were used (Figures $4 a$ and b). To confirm the above results, ECs that overexpress miR-126 were analyzed for Spred1 expression and Erk1/2 MAPK activation in response to oEVs. As reported in Figures $4 c$ and d, miR-126 overexpression in ECs led to Spred1 downregulation and Erk1/2 MAPK activation.

\section{nASC-derived EVs recapitulate oEV cargo after PA treatment}

Elevated non-esterified fatty acid (NEFA) plasma levels are commonly found in obese subjects. ${ }^{36}$ We therefore sought to determine whether high NEFA concentrations can account for the deranged oEV cargo. PA was used to treat nASC prior to EV recovery as it accounts for approximately $30 \%$ of total plasma NEFAs. No changes in EV number or size were detected under these experimental conditions (data not shown). VEGF, MMP-2 and miR-126 content were thus analyzed. The results reported in Figures $5 \mathrm{a}$ and $\mathrm{b}$ demonstrate that $\mathrm{EV}$ cargo was comparable to that of oEVs. Moreover, EVs that were released after PA treatment were again impaired in their ability to induce EC migration, tube-like formation and activate the Erk1/2 MAPK pathway (Figures $5 \mathrm{c}$ and e), when functional and biochemical experiments were performed. To further confirm our hypothesis, nASC treated with PA were analyzed for proliferation rate and senescence. Data reported in Supplementary Figures S3A and B demonstrate that PA-treated
nASCs mirror oASC features. Finally, the effect of HG treatment on EV functional capability was also evaluated, as diabetes is a part of natural history of obesity. EVs were recovered from oASCs that had undergone HG treatment and were evaluated for VEGF, MMP-2 and miR-126 content. We found that, while a significant reduction in miR-126 content was detected under these experimental conditions (Figure 5a), VEGF and MMP-2 content were not further reduced by $\mathrm{HG}$ treatment (Figure $5 \mathrm{~b}$ ). Moreover, HG treatment further impaired oEV ability to promote EC migration and tube-like structure formation (Figures $5 \mathrm{c}$ and e). No significant reduction in the number of viable cells was detected.

EVs recovered from the subcutaneous tissue of obese patients are also impaired in their pro-angiogenic potential

ASCs recovered from subcutaneous adipose tissue have been the object of the most frequent studies because of their regenerative capabilities. ${ }^{33}$ We therefore sought to investigate whether EVs that are released from these tissue-derived ASCs may be more efficient than oEVs. To this end, the effects of EVs released from subcutaneous tissue-derived ASCs of the same obese subjects (soEVs) and controls (snEVs) were evaluated. ASC stemness was validated by analyzing the expression of Oct4 (Figure 6a). We failed to detect differences in the number and size of sEVs in obese subjects and controls (Figures $6 \mathrm{~b}$ and $\mathrm{c}$ ). soEV biological activity was therefore evaluated and compared with that of snEVs. As reported in Figures $6 \mathrm{~d}$ and e, soEV treatment led to reduced EC migration, a significant reduction in tube-like structure formation and in the number and length of branches. No significant reduction in the number of viable cells was detected. Moreover, soEVs carried lower VEGF, MMP-2 and miR-126 content than snEVs (Figures $6 \mathrm{f}$ and $\mathrm{g}$ ). These data indicate that soEVs are also significantly impaired in their pro-angiogenic potential.

\section{DISCUSSION}

We have shown in the present study that EVs that are released from adipose tissue-derived ASCs of obese subjects are impaired in their pro-angiogenic potential. In particular, we have noticed that, unlike nEVs, oEVs bore reduced VEGF, MMP-2 and miR-126 content. This translates into reduced oEV capability to promote EC migration and tube-like structure formation. We also found that oEV impairment may depend on circulating levels of obesityassociated PA. These results indicate that obesity negatively impacts on ASC-derived EV functional capability and thus raises concerns as to their clinical use.

Regenerative medicine, and in particular cell-based therapy, has recently gained extensive attention, and various cell types, including ASCs, have been proposed for this role. ${ }^{3-5}$ The positive effects of ASC transplantation have recently been demonstrated to result from their ability to release trophic mediators (paracrine/ autocrine hypothesis) ${ }^{9,10}$ There is increasing evidence to suggest that ASC-secreted molecules, biologically present in the form of vesicles, may act as paracrine/endocrine mediators that are capable of modifying target cell functional capability. ${ }^{9,10}$ Vesicles, which have distinct biogenesis or cell origin, ${ }^{37}$ include exosomes, derived from the endosomal membrane compartment by exocytosis, and microvesicles generated by the budding of cell plasma membranes ${ }^{38}$ and are collectively denoted as EVs. EVs have recently been the focus of intense attention for their role as cell-to-cell communication mechanisms. ${ }^{13,14}$ In this regard, it has been reported that ASC-derived EVs are able to induce proangiogenic signals. ${ }^{17,33}$ The advantages of using EVs as an alternative therapeutic option with which to treat obesityrelated vascular damage have led us to investigate the potential clinical applications of obese-derived ASC EVs. We demonstrate that the number and size of EVs released from visceral tissuederived ASCs are comparable in obese and non-obese subjects. 

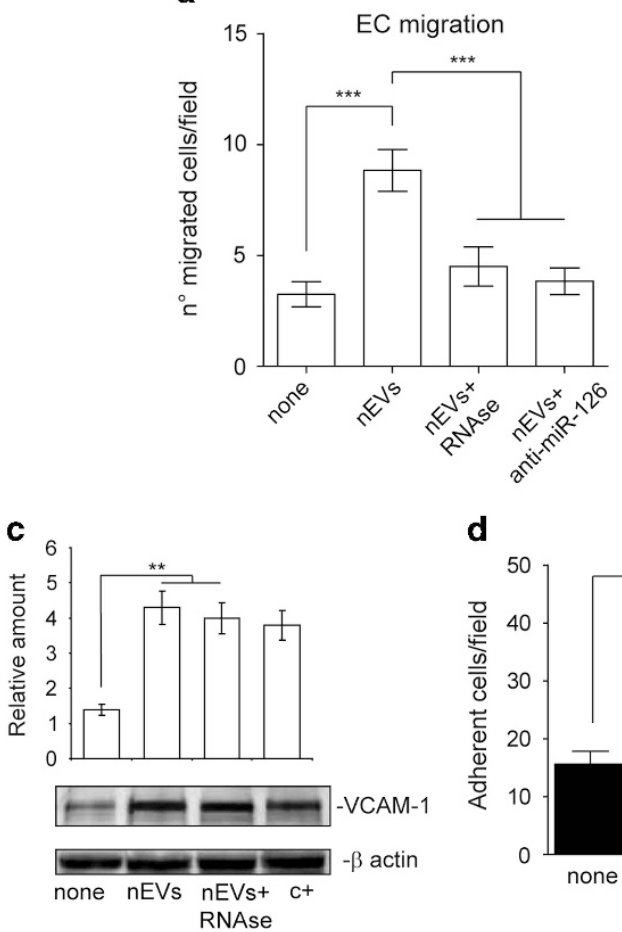

d

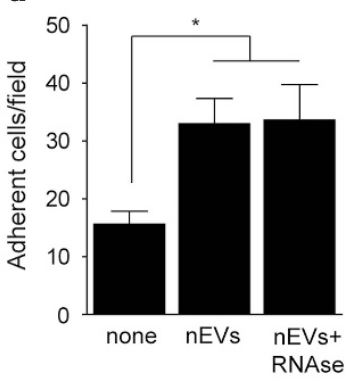

e EC migration

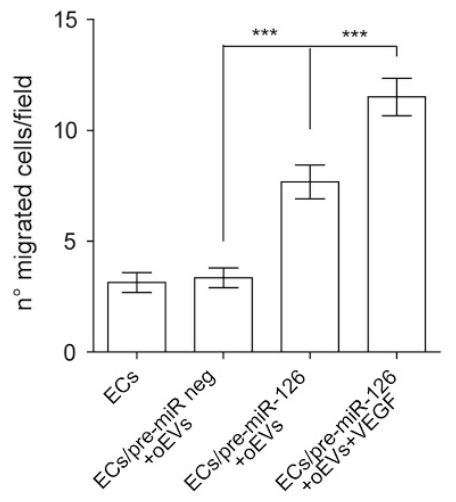

b
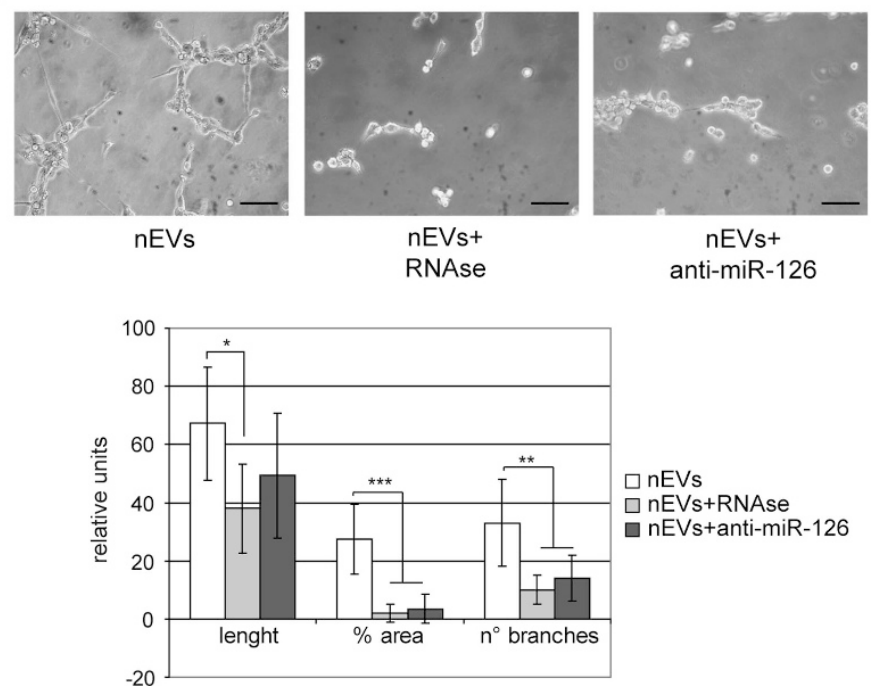

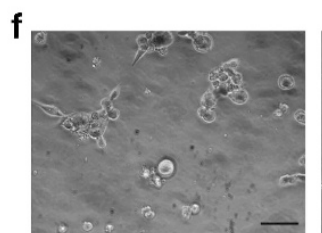

ECs/pre-miR neg

+ oEVs

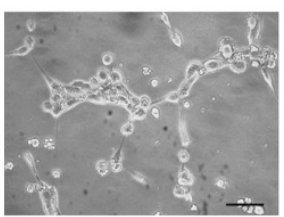

ECs/pre-miR-126 + oEVs

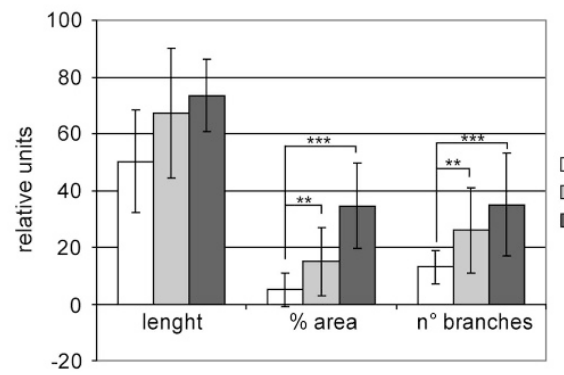

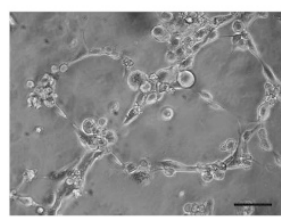

ECs/pre-miR-126 + oEVs + VEGF

Figure 3. EV-mediated miR-126 transfer is required for EV functional activities. (a) Migration assays performed on ECs treated with EVs recovered from nASCs that had either been treated with anti-miR-126 antagomir or nEVs+5U RNAse or not at all. The results are representative of five different experiments performed in triplicate $(n=5)\left({ }^{* * *} P<0.001 \mathrm{nEVs}\right.$ vs none; $n E V s+a n t i-m i R-126$ and $n E V s+5 U$ RNAse vs $n E V s$ ). (b) Representative photomicrographs of in vitro angiogenesis assays, showing tube-like structure formation by ECs treated as above. Quantitative analysis of the number and length of branches and percentage of vessel area (\% area) of in vitro formed vessel-like structures is reported as mean \pm s.e.m. The results are representative of four different experiments performed in triplicate $(n=4)$ (for length, ${ }^{*} P<0.05 \mathrm{nEVs}+\mathrm{RNAse}$ vs nEVs; for $\%$ area, ${ }^{* * *} P<0.001 \mathrm{nEVs}+\mathrm{RNAse}$ and nEVs+anti-miR-126 vs nEVs; for no. of branches, ${ }^{* *} P<0.01 \mathrm{nEVs}$ + RNAse and nEVs+anti-miR-126 vs nEVs). Scale bars indicate $50 \mu \mathrm{m}$. (c) Cell extracts from ECs treated with nEVs or nEVs+5U RNAse were analyzed for vascular cell adhesion molecule-1 (VCAM-1) content and normalized to $\beta$-actin content. IL-3-treated ECs were used as a positive control $(c+)$. The results are representative of five different experiments performed in triplicate $(n=5)(* * P<0.01 \mathrm{nEVs}$ and $\mathrm{nEVs}$ +5U RNAse vs none). (d) The adhesion assay was performed by plating red-labeled peripheral blood mononuclear cells on an EC monolayer that had been previously treated, or not, with $n E V s$ or $n E V s+5 U$ RNAse. Adherent cells were counted and reported as mean \pm s.e.m. per field ( $\times 20$ magnification). The results are representative of five different experiments performed in triplicate $(n=5)(* P<0.05 \mathrm{nEVs}$ and $\mathrm{nEVs}+5 \mathrm{U}$ RNAse vs none). (e) Migration assays performed on ECs, pretreated with pre-miR-126 precursor or pre-miR-negative control (premiR neg) and then stimulated with oEVs. EC migration was also evaluated on cells overexpressing miR-126 in the presence of oEVs and of VEGF $\left(10 \mathrm{ng} \mathrm{ml}^{-1}\right)$. The results are representative of four different experiments performed in triplicate $(n=4)(* * * P<0.001 \mathrm{ECs} / \mathrm{pre}-\mathrm{miR}-126$ +oEVs and ECs/pre-miR-126+oEVs+VEGF vs ECs/pre-miR neg+oEVs; ${ }^{* * *} P<0.001 \mathrm{ECs} /$ pre-miR-126+oEVs+VEGF vs ECs/pre-miR-126+oEVs). (f) Representative photomicrographs of in vitro angiogenesis assays performed on ECs treated as indicated in panel (c). Quantitative analysis of the number and length of branches and percentage of area of in vitro formed vessel-like structures is reported as mean \pm s.e.m. The results are representative of four different experiments performed in triplicate $(n=4)$ (for $\%$ area and no. of branches, $* * P<0.01$ ECs/pre-miR-126+oEVs vs ECs/pre-miR neg+oEVs, ${ }^{* * *} P<0.001$ ECs/pre-miR-126+oEVs+VEGF vs ECs/pre-miR neg+oEVs). Scale bars indicate $50 \mu \mathrm{m}$. 

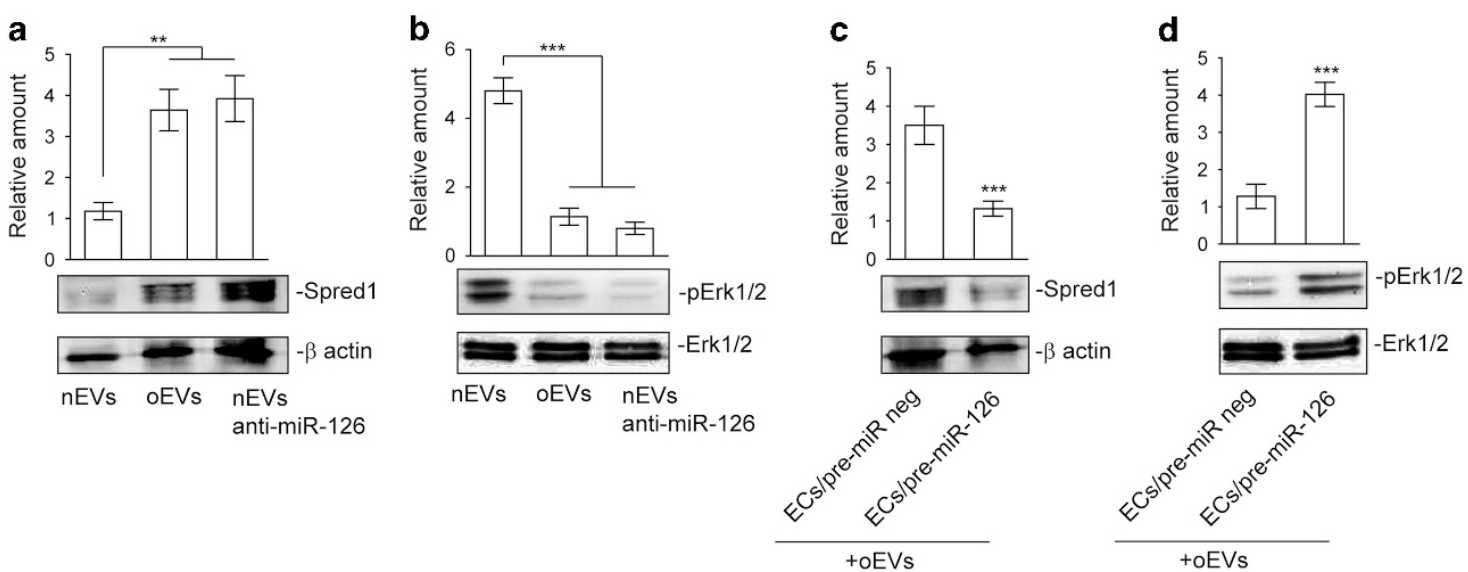

Figure 4. miR-126 oEV cargo drives Spred1 content and Erk1/2 activation in ECs. (a, b) Cell extracts from ECs treated with nEVs, oEVs or nEVs tanti-miR-126 were analyzed for Spred1 (a) and pErk1/2 (b) content, normalized to $\beta$-actin and Erk1/2 content, respectively. (**P $<0.01$ for Spred 1 and ${ }^{* * *} P<0.001$ for pErk1/2 oEVs and nEVs+anti-miR-126 vs nEVs, $n=4$ performed in triplicate). (c, d) ECs either pretreated with premiR-126 precursor or pre-miR-negative control (pre-miR neg) and then stimulated with oEVs were lysed and analyzed for Spred1 (c) and pErk1/2 (d) content. Protein level was normalized to $\beta$-actin and Erk1/2 content, respectively. The results are representative of four different experiments performed in triplicate $(n=4)(* * *<0.001 \mathrm{ECs} /$ pre-miR-126+oEVs vs ECs/pre-miR neg+oEVs).

a
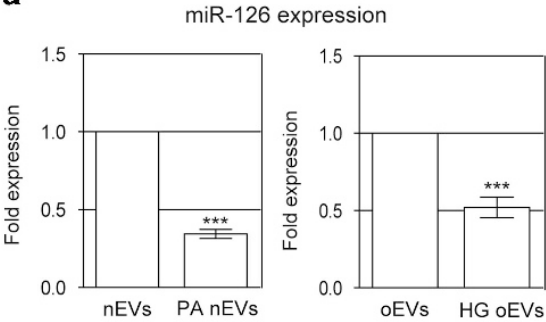

d

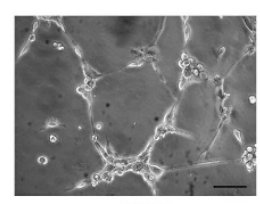

nEVs

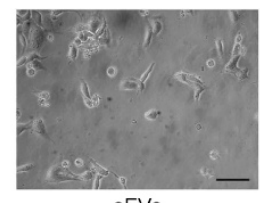

oEVs

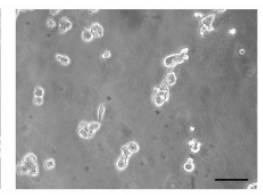

PA nEVs

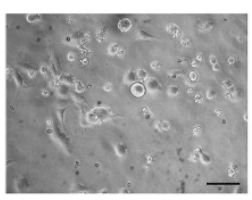

HG oEVs

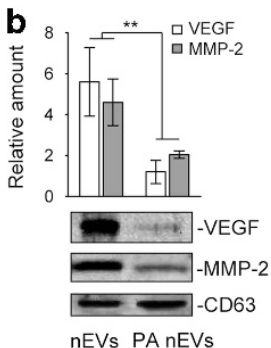

nEVs PA nEVs

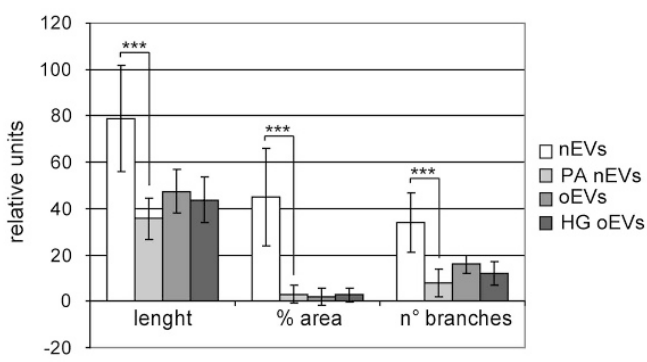

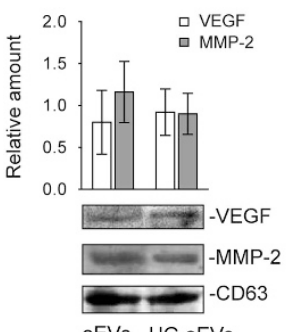

oEVs HG oEVs

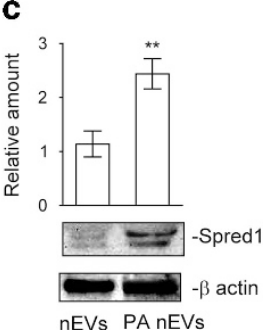

nEVs PAnEVs

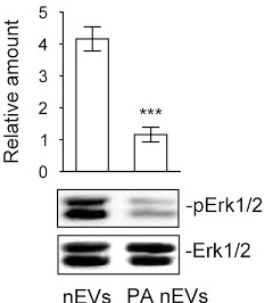

nEVs PA nEVs

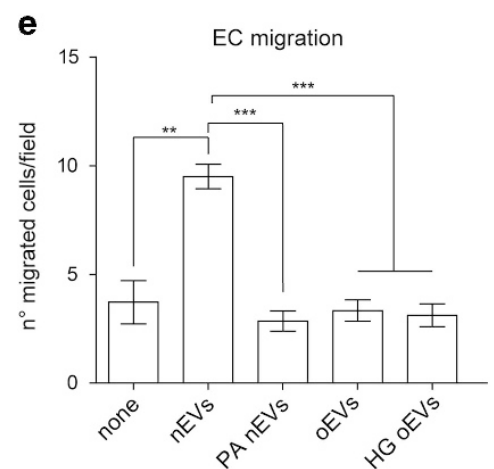

Figure 5. EVs derived from PA-treated $n A S C s$ recapitulate oEV cargo. (a) miR-126 expression was evaluated by quantitative real-time $P C R$ either on $n E V s$ and $n E V s$ recovered from nASC pretreated with PA $\left(300 \mu \mathrm{mol} I^{-1}\right)$, left panel, or on oEVs and oEVs recovered from oASC pretreated with HG $\left(25 \mathrm{mmol} \mathrm{I}^{-1}\right)$. Data normalized to RNU6B are representative of four different experiments performed in triplicate $(n=4)$. $(* * * P<0.001$ PA nEVs vs nEVs; HG oEVs vs oEVs). (b) nEVs, PA nEVs, oEVs and HG oEVs, obtained as described above, were analyzed for VEGF and MMP-2 content and normalized to CD63 content. ( ${ }^{* *} P<0.01$ PA nEVs vs nEVs for VEGF and MMP-2, $n=4$ performed in triplicate). (c) Cell extracts from ECs treated with nEVs or PA nEVs were analyzed for Spred 1 and pErk1/2 content. The protein level was normalized to $\beta$-actin and Erk1/2 content, respectively. ( ${ }^{* *} P<0.01$ PA nEVs vs $\mathrm{nEVs}$ for Spred1; ${ }^{* *} P<0.001 \mathrm{nEVs}$ vs $\mathrm{nEVs}$ for pErk1/2, $n=4$ performed in triplicate). (d) Representative photomicrographs of in vitro angiogenesis assays, showing tube-like structure formation by ECs treated with $\mathrm{nEV}$, PA $\mathrm{nEV}$, oEVs or HG oEVs. Quantitative analysis of the number and length of branches and percentage of vessel area (\% area) of in vitro formed vessellike structures is reported as mean \pm s.e.m. The results are representative of four different experiments $(n=4)$ performed in triplicate (for length, ${ }^{* * *} P<0.001$ PA nEVs vs nEVs; for $\%$ area, ${ }^{* * *} P<0.001$ PA nEVs vs nEVs; for no. of branches, ${ }^{* * *} P<0.001$ PA nEVs vs nEVs). Scale bars indicate $50 \mu \mathrm{m}$. (e) Migration assays performed on ECs untreated or treated as indicated. The results are representative of four different experiments $(n=4)$ performed in triplicate $\left({ }^{* *} P<0.01\right.$ nEVs vs none; ${ }^{* *} P<0.001$ PA nEVs vs nEVs; ${ }^{* * *} P<0.001$ oEVs and HG oEVs vs nEVs).

In addition, we have found that both oEVs and nEVs are able to cross the plasma membrane and to release their cargo within target cells, ECs. However, functional studies demonstrate that oEVs are biologically inactive, as they were unable to induce EC migration and tube-like structure formation. The EV mechanism of action is known to rely on their ability to transfer functional proteins and RNAs to target cells. ${ }^{14}$ A number of proteins and miRs are carried by EVs, including those that participate in the angiogenic process. $^{39}$ VEGF, MMP-2 and miR-126 content was found to be lower in oEVs than in nEVs, and this is consistent with 
a

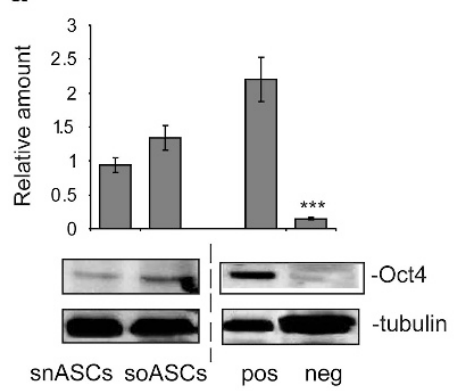

d

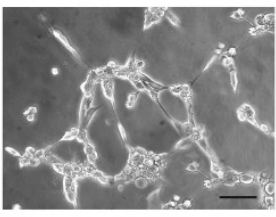

snEVs

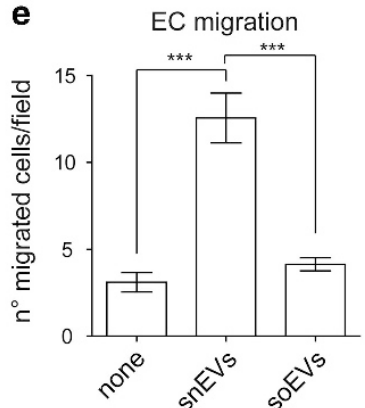

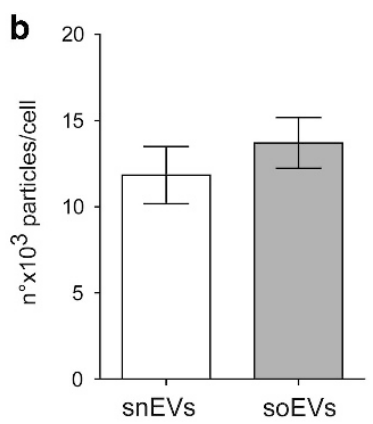

C

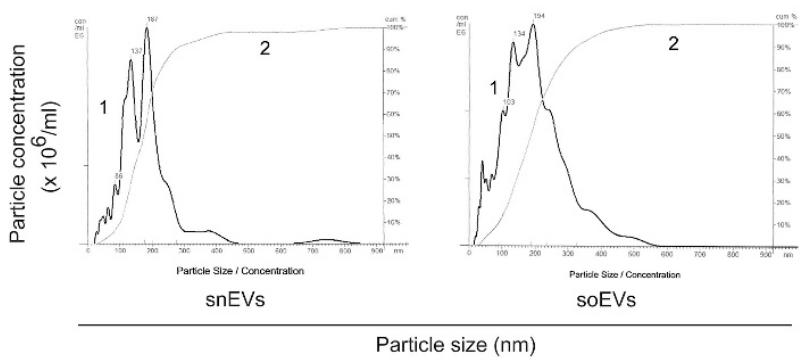

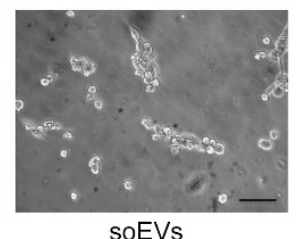

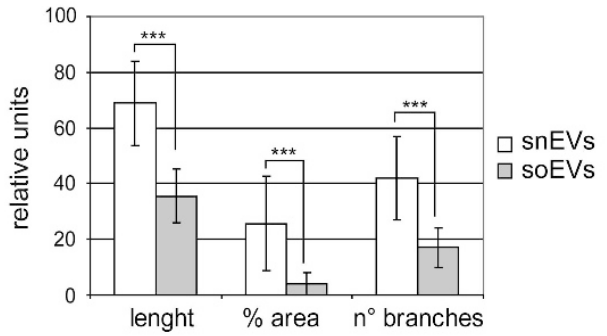

f

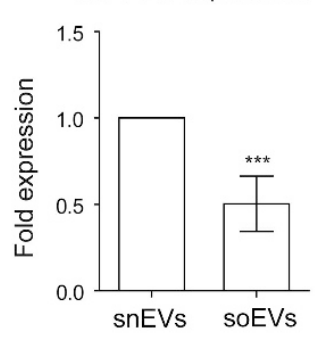

g

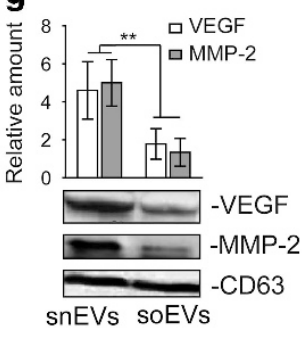

Figure 6. EVs recovered from the subcutaneous tissue of obese patients are impaired in their pro-angiogenic potential as well. (a) Representative Oct4 content obtained from cell extracts of subcutaneous-derived ASCs ( $s n A S C s, n=6 ;$ soASCs, $n=10$ ). Protein levels were normalized to tubulin content. pos: positive control, human ASCs purchased from Lonza; neg: negative control, adipocytes derived from the $\mathrm{NIH} 3 \mathrm{~T} 3 \mathrm{~L} 1$ cell line) (***P<0.001 neg vs pos control). (b) Representative images of NanoSight analyses performed on the $100 \mathrm{k}$ fraction of subcutaneous ASC-derived EVs. Curve 1, relationship between particle distribution (left $y$ axis) and particle size ( $x$ axis); curve 2, correlation between cumulative percentage distribution of particles (percentile in right $y$ axis) and particle size ( $x$ axis). (c) Number of EV particles (mean \pm s.e.m.) per cell at isolation. Data refer to EVs from subcutaneous adipose tissue samples recovered from obese subjects $($ soEVs, $n=10$ ) and non-obese subjects (snEVs, $n=6$ ). (d) Representative photomicrographs of in vitro angiogenesis assays, showing tube-like structure formation by ECs treated with snEVs or soEVs. Quantitative analysis of the number and length of branches and percentage of vessel area (\% area) of in vitro formed vessel-like structures is reported as mean \pm s.e.m. The results are representative of four different experiments $(n=4)$ performed in triplicate (for length, ${ }^{* * *} P<0.001$ soEVs vs snEVs; for $\%$ area, ${ }^{* * *} P<0.001$ soEVs vs snEVs; for no. of branches, ${ }^{* * *} P<0.001$ soEVs vs snEVs). Scale bars indicate $50 \mu \mathrm{m}$. (e) Migration assays performed on ECs untreated or treated with snEVs or soEVs. The results are representative of four different experiments $(n=4)$ performed in triplicate ( ${ }^{* * *} P<0.001$ snEVs vs none; ${ }^{* * *} P<0.001$ soEVs vs snEVs). (f) miR126 expression was evaluated by quantitative real-time PCR on snEVs and soEVs. Data normalized to RNU6B are representative of all samples (snEVs, $n=6$; soEVs, $n=10$ ) ( ${ }^{* * *} P<0.001$ soEVs vs snEVs). (g) VEGF and MMP-2 content was evaluated in snEVs and soEVs. Protein levels were normalized to CD63 content. ( ${ }^{*} P<0.01$ soEVs, $n=10$, vs snEVs, $n=6$ ).

the functional studies. Moreover, gain- and loss-of-function experiments have shown that miR-126 is necessary if EVs are to trigger angiogenic signals via Spred1 downregulation and Erk1/2 MAPK pathway activation. Finally, the finding that the addition of VEGF to ECs that overexpress miR-126 was able to almost completely restore migration and tube-like structure formation further sustains the role that EV cargo has in mediating their biological actions.

Obesity is associated with a chronic elevation of NEFAs, and PA is one of the most abundant circulating NEFAs in obese patients. ${ }^{36}$ PA has been shown to act as an endogenous signaling molecule in various cellular contexts. ${ }^{25}$ In particular, PA has recently been linked to obesity-associated neurodegenerative diseases. ${ }^{40} \mathrm{We}$ herein provide evidence to suggest that elevated PA concentrations can induce changes in ASC-derived EV cargo and that this event translates into impaired EV-mediated pro-angiogenic signal activation. Moreover, in a reflection of results in oEVs, EVs released by ASCs from non-obese subjects that were challenged with PA carried reduced VEGF, MMP-2 and miR-126 content. This suggests that increased PA concentrations in obesity may be relevant in changing ASC-derived EV cargo.

Alternatively, it is conceivable to hypothesize that oEV content reflects the cellular phenotype changes occurring in obese patients. In fact, the microenvironment has been shown to modify both EV miR and protein profile. ${ }^{41,42}$ Consistent with this possibility, our PA experiments suggest that altering nASC lipid composition modifies EV cargo and functions. Comparative lipidomic analysis of nASCs and oASCs EVs, not evaluated in this study, might provide further explanations for the observed different biological activities of EVs.

Obesity is a leading risk factor for the development of type 2 diabetes, and $>80 \%$ of adults who suffer from type 2 diabetes are 
overweight or obese. ${ }^{43}$ Thus, although our obese subjects were not diabetics, we sought to determine whether a diabetic milieu may further impair EV bioactivity by changing their cargo. Mocharla et al. ${ }^{44}$ and Jansen et al. ${ }^{45}$ have shown reduced miR-126 content in EVs released by CD34+ peripheral blood mononuclear cells and ECs in a diabetic setting. As stated above, we have noticed that obese-derived ASCs release EVs that are already impaired in their miR-126 content and that the hyperglycaemic milieu additionally harms miR-126 cargo and EV bioactivity.

Easy access by minimally invasive methods means that subcutaneous adipose tissue is of special interest as it is an abundant source of ASC-derived EVs that can be exploited in regenerative medicine. ${ }^{10,11}$ As a matter of fact, the pro-angiogenic potential of EVs released by subcutaneous tissue-derived ASCs has been reported. ${ }^{17,34}$ This is not the case for EVs released from the subcutaneous adipose tissue-derived ASCs of obese patients. Indeed, they share a similar cargo and impaired bioactivity with oEVs.

Despite the exciting results of cell-based approaches in several clinical settings, ${ }^{46}$ the technique's true clinical impact is still open to debate. The intrinsic features of EVs may make them suitable ASC derivates for use in regenerative medicine. ${ }^{10,11}$ EVs also show advantages over cell therapy as they are biocompatible and do not expose patients to the risk of cell maldifferentiation. ${ }^{11}$ A general strategy for ASC-derived EV clinical use has been proposed by Katsuda et al. $^{47}$ However, it is necessary for the molecules that underlie ASC-EV-mediated therapeutics to be identified if we are to realize full clinical application. Our data support the notion that miRs, and in particular EV miR-126 content and miR126's target, impact on EV pro-angiogenic properties, which is in agreement with miR screening and gene ontology target analysis results. ${ }^{11,48}$

There is a need to study EVs in different pathological contexts as it will furnish important information as to their potential therapeutic applications in regenerative medicine. EVs would appear to possess all the potential attributed to cell-based medicines. However, we herein provide evidence which suggests that the potential damaging effects of environmental cues must be always taken into account if ASC-derived EV clinical applications are to be pursued.

\section{CONFLICT OF INTEREST}

The authors declare no conflict of interest.

\section{ACKNOWLEDGEMENTS}

Professor MF Brizzi is the guarantor of this work, had full access to all data and takes full responsibility for data integrity and the accuracy of data analysis. This work has been supported by grants obtained by MFB and AS from the Fondazione per la Ricerca Diabetologica FO.Ri.SID.

\section{REFERENCES}

1 Imes CC, Burke LE. The obesity epidemic: the United States as a cautionary tale for the rest of the world. Curr Epidemiol Rep 2014; 1: 82-88.

2 Lepor NE, Fouchia DD, McCullough PA. New vistas for the treatment of obesity: turning the tide against the leading cause of morbidity and cardiovascular mortality in the developed world. Rev Cardiovasc Med 2013; 14: 20-39.

3 Zuk PA, Zhu M, Ashjian P, De Ugarte DA, Huang J, Mizuno H et al. Human adipose tissue is a source of multipotent stem cells. Mol Biol Cell 2002; 13: 4279-4295.

4 Gimble JM, Katz AJ, Bunnell BA. Adipose derived stem cells for regenerative medicine. Circ Res 2007; 100: 1249-1260.

5 Schaffler A, Buchler C. Concise review: adipose tissue-derived stromal cells--basic and clinical implications for novel cell-based therapies. Stem Cells 2007; 25: 818-827.

6 Dentelli P, Barale C, Togliatto G, Trombetta A, Olgasi C, Gili M et al. A diabetic milieu promotes OCT4 and NANOG production in human visceral-derived adipose stem cells. Diabetologia 2013; 56: 173-184.
7 Kokai LE, Marra K, Rubin JP. Adipose stem cells: biology and clinical applications for tissue repair and regeneration. Transl Res 2014; 163: 399-408.

8 Kono TM, Sims EK, Moss DR, Yamamoto W, Ahn G, Diamond J et al. Human adipose-derived stromal/stem cells protect against STZ-induced hyperglycemia: analysis of hASC-derived paracrine effectors. Stem Cells 2014; 32: 1831-1842.

9 Ratajczak J, Kucia M, Mierzejewska K, Marlicz W, Pietrzkowski Z, Wojakowski W et al. Paracrine proangiopoietic effects of human umbilical cord blood-derived purified CD133+ cells--implications for stem cell therapies in regenerative medicine. Stem Cells Dev 2013; 22: 422-430.

10 Ratajczak MZ. A novel view of the adult bone marrow stem cell hierarchy and stem cell trafficking. Leukemia 2014; 29: 776-782.

11 Quesenberry PJ, Goldberg LR, Aliotta JM, Dooner MS, Pereira MG, Wen S et al. Cellular phenotype and extracellular vesicles: basic and clinical considerations. Stem Cells Dev 2014; 23: 1429-1436.

12 De Jong OG, Van Balkom BW, Schiffelers RM, Bouten CV, Verhaar MC. Extracellular vesicles: potential roles in regenerative medicine. Front Immunol 2014; 5: 608.

13 Record M, Subra C, Silvente-Poirot S, Poirot M. Exosomes as intercellular signalosomes and pharmacological effectors. Biochem Pharmacol 2011; 81: 1171-1182.

14 Tetta C, Ghigo E, Silengo L, Deregibus MC, Camussi G. Extracellular vesicles as an emerging mechanism of cell-to-cell communication. Endocrine 2013; 44: 11-19.

15 Eirin A, Riester SM, Zhu XY, Tang H, Evans JM, O'Brien D et al. MicroRNA and mRNA cargo of extracellular vesicles from porcine adipose tissue-derived mesenchymal stem cells. Gene 2014; 551: 55-64.

16 Mause SF, Weber C. Microparticles: protagonists of a novel communication network for intercellular information exchange. Circ Res 2010; 107: 1047-1057.

17 Ranghino A, Cantaluppi V, Grange C, Vitillo L, Fop F, Biancone L et al. Endothelial progenitor cell-derived microvesicles improve neovascularization in a murine model of hindlimb ischemia. Int J Immunopathol Pharmacol 2012; 25: 75-85.

18 Cantaluppi V, Gatti S, Medica D, Figliolini F, Bruno S, Deregibus MC et al. Microvesicles derived from endothelial progenitor cells protect the kidney from ischemia-reperfusion injury by microRNA-dependent reprogramming of resident renal cells. Kidney Int 2012; 82: 412-427.

19 Bruno S, Grange C, Collino F, Deregibus MC, Cantaluppi V, Biancone L et al. Microvesicles derived from mesenchymal stem cells enhance survival in a lethal model of acute kidney injury. PLoS One 2012; 7: e33115.

20 Herrera MB, Fonsato V, Bruno S, Grange C, Gilbo N, Romagnoli R et al. Human liver stem cells improve liver injury in a model of fulminant liver failure. Hepatology 2013; 57: 311-319.

21 Bruno S, Collino F, Tetta C, Camussi G. Dissecting paracrine effectors for mesenchymal stem cells. Adv Biochem Eng Biotechnol 2013; 129: 137-152.

22 Colombo M, Raposo G, Théry C. Biogenesis, secretion, and intercellular interactions of exosomes and other extracellular vesicles. Annu Rev Cell Dev Biol 2014; 30: $255-289$

23 Loyer X, Vion AC, Tedgui A, Boulanger CM. Microvesicles as cell-cell messengers in cardiovascular diseases. Circ Res 2014; 114: 345-353.

24 Wang Y, Chen LM, Liu ML. Microvesicles and diabetic complications--novel mediators, potential biomarkers and therapeutic targets. Acta Pharmacol Sin 2014; 35: 433-443.

25 Trombetta A, Togliatto G, Rosso A, Dentelli P, Olgasi C, Cotogni P et al. Increase of palmitic acid concentration impairs endothelial progenitor cell and bone marrowderived progenitor cell bioavailability: role of the STAT5/PPARY transcriptional complex. Diabetes 2013; 62: 1245-1257.

26 Togliatto G, Trombetta A, Dentelli P, Rosso A, Brizzi MF. MIR221/MIR222-driven post-transcriptional regulation of P27KIP1 and P57KIP2 is crucial for high-glucoseand AGE-mediated vascular cell damage. Diabetologia 2011; 54: 1930-1940.

27 Deregibus MC, Cantaluppi V, Calogero R, Lo lacono M, Tetta C, Biancone L et al. Endothelial progenitor cell derived microvesicles activate an angiogenic program in endothelial cells by a horizontal transfer of mRNA. Blood 2007: 110: 2440-2448.

28 Lopatina T, Bruno S, Tetta C, Kalinina N, Porta M, Camussi G. Platelet-derived growth factor regulates the secretion of extracellular vesicles by adipose mesenchymal stem cells and enhances their angiogenic potential. Cell Commun Signal 2014; 12: 26.

29 Fonsato V, Collino F, Herrera MB, Cavallari C, Deregibus MC, Cisterna B et al. Human liver stem cell-derived microvesicles inhibit hepatoma growth in SCID mice by delivering antitumor microRNAs. Stem Cells 2012; 30: 1985-1998.

30 Olgasi C, Dentelli P, Rosso A, lavello A, Togliatto G, Toto V et al. DNA vaccination against membrane-bound Kit ligand: a new approach to inhibiting tumour growth and angiogenesis. Eur J Cancer 2014; 50: 234-246.

31 Togliatto G, Trombetta A, Dentelli P, Gallo S, Rosso A, Cotogni P et al. Unacylated ghrelin (UnAG) induces oxidative stress resistance in a glucose intolerance mouse model and peripheral artery disease by restoring endothelial cell miR-126 expression. Diabetes 2015; 64: 1370-1382.

32 Baglioni S, Francalanci M, Squecco R, Lombardi A, Cantini G, Angeli R et al. Characterization of human adult stem-cell populations isolated from visceral and subcutaneous adipose tissue. FASEB J 2009; 23: 3494-3505. 
33 Schreml S, Babilas P, Fruth S, Orsó E, Schmitz G, Mueller MB et al. Harvesting human adipose tissue-derived adult stem cells: resection versus liposuction. Cytotherapy 2009; 11: 947-957.

34 Dignat-George F, Boulanger CM. The many faces of endothelial microparticles. Arterioscler Thromb Vasc Biol 2011; 31: 27-33.

35 Wang S, Aurora AB, Johnson BA, Qi X, McAnally J, Hill JA et al. The endothelialspecific microRNA miR-126 governs vascular integrity and angiogenesis. Dev Cell 2008; 15: 261-271.

36 Rauschert S, Uhl O, Koletzko B, Hellmuth C. Metabolomic biomarkers for obesity in humans: a short review. Ann Nutr Metab 2014; 64: 314-324.

37 György B, Szabó TG, Pásztói M, Pál Z, Misják P, Aradi B et al. Membrane vesicles, current state-of-the-art: emerging role of extracellular vesicles. Cell Mol Life Sci 2011; 68: 2667-2688.

38 Ostrowski M, Carmo NB, Krumeich S, Fanget I, Raposo G, Savina A et al. Rab27a and Rab27b control different steps of the exosome secretion pathway. Nat Cell Biol 2010; 12: 19-30.

39 Gangoda L, Boukouris S, Liem M, Kalra H, Mathivanan S. Extracellular vesicles including exosomes are mediators of signal transduction: Are they protective or pathogenic? Proteomics 2014; 15: 260-271.

$40 \mathrm{Hsiao} \mathrm{YH}$, Lin $\mathrm{Cl}$, Liao H, Chen YH, Lin SH. Palmitic acid-induced neuron cell cycle $\mathrm{G} 2 / \mathrm{M}$ arrest and endoplasmic reticular stress through protein palmitoylation in SH-SY5Y human neuroblastoma cells. Int J Mol Sci 2014; 15: 20876-20899.

41 Colombo M, Raposo G, Théry C. Biogenesis, secretion, and intercellular interactions of exosomes and other extracellular vesicles. Annu Rev Cell Dev Biol 2014; 30: 255-289.

42 Kucharzewska $P$, Belting $M$. Emerging roles of extracellular vesicles in the adaptive response of tumour cells to microenvironmental stress. J Extracell Vesicles 2013; 2: doi:10.3402/jev.v2i0.20304.
43 Al-Goblan AS, Al-Alfi MA, Khan MZ. Mechanism linking diabetes mellitus and obesity. Diabetes Metab Syndr Obes 2014; 7: 587-591.

44 Mocharla P, Briand S, Giannotti G, Dörries C, Jakob P, Paneni F et al. AngiomiR-126 expression and secretion from circulating CD34(+) and CD14(+) PBMCs: role for proangiogenic effects and alterations in type 2 diabetics. Blood 2013; 121: 226-236.

45 Jansen F, Yang X, Hoelscher M, Cattelan A, Schmitz T, Proebsting S et al. Endothelial microparticle-mediated transfer of MicroRNA-126 promotes vascular endothelial cell repair via SPRED1 and is abrogated in glucose-damaged endothelial microparticles. Circulation 2013; 128: 2026-2038.

46 Simerman AA, Dumesic DA, Chazenbalk GD. Pluripotent muse cells derived from human adipose tissue: a new perspective on regenerative medicine and cell therapy. Clin Transl Med 2014; 3: 12.

47 Katsuda T, Kosaka N, Takeshita F, Ochiya T. The therapeutic potential of mesenchymal stem cell-derived extracellular vesicles. Proteomics 2013; 13: 1637-1653.

48 Lindoso RS, Collino F, Bruno S, Araujo DS, Sant'Anna JF, Tetta C et al. Extracellular vesicles released from mesenchymal stromal cells modulate miRNA in renal tubular cells and inhibit ATP depletion injury. Stem Cells Dev 2014; 23: 1809-1819.

(c) (i) $(\Theta$ This work is licensed under a Creative Commons Attributioncc. NonCommercial-NoDerivs 4.0 International License. The images or other third party material in this article are included in the article's Creative Commons license, unless indicated otherwise in the credit line; if the material is not included under the Creative Commons license, users will need to obtain permission from the license holder to reproduce the material. To view a copy of this license, visit http:// creativecommons.org/licenses/by-nc-nd/4.0/

Supplementary Information accompanies this paper on International Journal of Obesity website (http://www.nature.com/ijo) 